CERN-EP / 2000-112

10 August 2000

\title{
Charge collection efficiency of an irradiated cryogenic double-p silicon detector
}

\author{
RD39 Collaboration: \\ K. Borer and S. Janos \\ LHEP, Sidlerstrasse 5, CH-3012 Bern, Switzerland \\ Z. Li and B. Dezillie \\ Brookhaven National Laboratory, Upton, NY 11973-5000, USA \\ C. Da Viá and V. Granata ${ }^{1}$ \\ University of Brunel, Uxbridge, Middlesex UB8 3PH, UK
}

L. Casagrande*, R. W. I. de Boer ${ }^{2}$, C. Lourenço, T. O. Niinikoski and V. G. Palmieri CERN, CH-1211 Geneva, Switzerland

S. Chapuy, Z. Dimcovski and E. Grigoriev

Department de Radiologie, Université de Genève, CH-1211 Geneva, Switzerland

W. Bell, S.R.H. Devine, G. Ruggiero', V. O'Shea and K. Smith

Department of Physics and Astronomy, University of Glasgow, Glasgow G12 8QQ, UK

$$
\text { P. Berglund }
$$

Low Temperature Laboratory, Helsinki University of Technology, FI-02150 Espoo, Finland

W. de Boer, F. Hauler, S. Heising and L. Jungermann

IEKP University of Karlsruhe, D-76128 Karlsruhe, Germany

M. Abreu, P. Rato and P. Sousa

LIP, av. E. Garcia, P-1000 Lisbon, Portugal

V. Cindro, M. Mikuz and M. Zavrtanik

Jozef Stefan Institute, Exp. Particle Physics Dep., PO Box 3000, 1001 Ljubljana, Slovenia

A. Esposito and S. Paul

Physik Department E18, Technische Universität München, D-85748 Garching, Germany

S. Buontempo, N. D'Ambrosio and S. Pagano

Universitá Frederico II di Napoli, Dipartimento di Fisica and INFN, Napoli, Italy

V. Eremin and E. Verbitskaya

Ioffe Physico-Technical Inst., Russian Academy of Sciences, St. Petersburg 194021, Russia

Submitted to Nucl. Instr. and Meth. In Phys. Res. A

\footnotetext{
${ }^{1}$ Also at CERN.

* Corresponding author: Luca.Casagrande@cern.ch.

${ }^{2}$ Visitor from Eindhoven University of Technology.
} 


\section{Abstract}

We present results on the measurement of the charge collection efficiency of a $\mathrm{p}^{+} / \mathrm{n} / \mathrm{p}^{+}$silicon detector irradiated to $1 \times 10^{15} \mathrm{n} / \mathrm{cm}^{2}$, operated in the temperature range between $80 \mathrm{~K}$ and 200 K. For comparison, measurements obtained with a standard silicon diode $\left(\mathrm{p}^{+} / \mathrm{n} / \mathrm{n}^{+}\right)$, irradiated to the same fluence, are also presented. Both detectors show a dramatic increase of the CCE when operated at temperatures around $130 \mathrm{~K}$. The double-p detector shows a higher CCE regardless of the applied bias and temperature, besides being symmetric with respect to the polarity of the bias voltage. At $130 \mathrm{~K}$ and $500 \mathrm{~V}$ applied bias the double-p detector shows a CCE of $80 \%$, an unprecedented result for a silicon detector irradiated to such a high dose. 


\section{Introduction}

The "Lazarus effect", described as the charge collection efficiency (CCE) recovery of heavily irradiated silicon detectors at cryogenic temperatures, was observed for the first time in 1998 [1]. Since then, the CERN RD39 Collaboration [2] has carried out a systematic study of irradiated silicon pad [3] and microstrip [4] detectors for a deeper understanding of this phenomenon.

Radiation creates defects in the silicon lattice that appear as deep energy levels in the forbidden band gap [5]. These defects can trap charge carriers and thus contribute to the effective doping concentration, $N_{\text {eff. }}$ At very high doses, this generates a dramatic increase of the full depletion voltage $\left(V_{\mathrm{fd}}\right)$, and eventually, when $V_{\mathrm{fd}}$ is greater than the highest possible bias voltage, the detector cannot be reliably operated any longer [6]. Furthermore, charge carriers generated by a particle traversing the detector may be trapped in the radiation-induced defects. If the de-trapping process happens on a time scale that is comparable to or larger than the detector readout time, trapping causes a fraction of the signal to be lost $[6,7]$.

At cryogenic temperatures, the de-trapping process is strongly reduced and carriers stay at the radiation-induced defects. Under these circumstances, many traps are constantly filled and thus inactive. The charge carriers created by a particle traversing the detector cannot be trapped and therefore no signal is lost. Moreover, the effective doping concentration, $N_{\text {eff }}$, becomes less negative, which results in a decrease of the depletion voltage [8]. This means that for the same applied voltage the active thickness of the detector, and therefore the charge collected at its electrodes, is larger at cryogenic temperatures than at or near room temperature.

Before this work, the Lazarus effect was investigated only on conventional $\mathrm{p}^{+} / \mathrm{n} / \mathrm{n}^{+}$silicon detectors. In this case, before irradiation the junction depletes from the $\mathrm{p}^{+}$-side. When irradiated beyond type inversion, such a structure still behaves like a diode, except that the junction now depletes from the $\mathrm{n}^{+}$-side, as depicted in Figure 1 top. It was found [3] that the CCE of standard diodes reaches a maximum value around $130 \mathrm{~K}$, for all investigated samples regardless of the fluence to which they were irradiated. When operated in reverse bias, the CCE degrades with time till it reaches a stable value. For example, in the case of a sample irradiated up to $1 \times 10^{15} \mathrm{n} / \mathrm{cm}^{2}$, a stable CCE of $\sim 20 \%$ is achieved with $250 \mathrm{~V}$ reverse bias at $\mathrm{T}=130 \mathrm{~K}$.

Due to the high bulk resistivity, which considerably limits the current, reliable forward bias operation becomes possible at cryogenic temperatures. In this case, the CCE does not 
evolve with time and it stays at its initial maximum value. Thus a CCE, higher by a factor of 2 to 3 compared with the value obtained in reverse bias, can be achieved in forward bias [3]. These effects were predicted in [9] and were already observed in the case of moderate cooling [10]. It is worth stressing that in the case of silicon detectors, irradiated up to $1 \times 10^{15} \mathrm{n} / \mathrm{cm}^{2}$ and operated under forward bias at $\mathrm{T}=-18^{\circ} \mathrm{C}$, a current of the order of $\sim 6 \mu \mathrm{A} / \mathrm{mm}^{2}$ is observed, which is too large and limits the application only to finely segmented devices [10].

In order to avoid the time dependence, one could think of using a diode in reverse bias untill the resistivity of the bulk, which increases with the accumulated dose, is large enough to enable reliable operation under forward bias. Unfortunately this approach has the unpractical drawback that bipolar electronics must be used for biasing and to read out the detector signal. An alternative solution would consist of using ohmic devices. Of course, this is only possible at cryogenic temperatures, where the steady state current of the device is small enough to make the signal to background ratio acceptable.

This work was dedicated to the investigation of a $\mathrm{p}^{+} / \mathrm{n} / \mathrm{p}^{+}$implanted silicon detector, hereafter named as "double-p". Such a device can be considered as two diodes in opposite directions (see Figure 1 bottom). The detector never conducts whatever the polarity of the bias voltage is because there is always one diode in reverse bias. After type inversion, one would expect "ohmic-like" behaviour, and thus its CCE should be symmetric for positive and negative bias and it should not feature any time dependence. In this work, the CCE behaviour of a double-p detector irradiated to $1 \times 10^{15} \mathrm{n} / \mathrm{cm}^{2}$ was investigated in detail in the temperature range between $80 \mathrm{~K}$ and $200 \mathrm{~K}$. Results of the measurements on the double-p detector are compared to the ones obtained with a standard silicon diode $\left(\mathrm{p}^{+} / \mathrm{n} / \mathrm{n}^{+}\right)$irradiated to the same dose.

\section{Samples and experimental set-up}

The investigated double-p detector was an $\mathrm{Al} / \mathrm{p}^{+} / \mathrm{n} / \mathrm{p}^{+} / \mathrm{Al}$ implanted silicon pad detector fabricated at Brookhaven National Laboratory. The sample thickness was $400 \mu \mathrm{m}$ and its resistivity before irradiation was $4-6 \mathrm{k} \Omega \mathrm{cm}$. For comparison, we have also investigated a standard $\mathrm{Al} / \mathrm{p}^{+} / \mathrm{n} / \mathrm{n}^{+} / \mathrm{Al}$ implanted silicon detector, with the same properties. Both samples had a sensitive area of $7 \times 7 \mathrm{~mm}^{2}$, surrounded by a guard ring. The structures were manufactured using a simple processing technology involving only three mask steps. 
The detectors were irradiated with neutrons at the TRIGA nuclear reactor in Ljubljana. The fluence was determined by gold activation converted to non-ionising energy loss in silicon, calculated from the measured neutron energy spectra and using the damage functions from [11] and [12]. For both samples, the total dose was $1 \times 10^{15} 1 \mathrm{MeV}$ neutrons per $\mathrm{cm}^{2}$ $\left(\mathrm{n} / \mathrm{cm}^{2}\right)$, which exceeds the type inversion threshold. The systematic error on the fluence is about $10 \%$. During and after irradiation the detectors were kept at room temperature. It is worth stressing that annealing effects have been shown [3] to have no significant influence on the CCE of silicon detectors when they are operated at cryogenic temperatures.

The measurements described in this paper were performed in a simple cryostat that was specially designed and built for easy and quick CCE measurements of silicon detectors. It consists of an outer vacuum vessel of $100 \mathrm{~mm}$ diameter, and an inner vessel, which acts as liquid nitrogen reservoir. The two vessels are mechanically connected by a top flange that provides ports with hermetic feed-through for the detector and instrumentation connections. Figure 2 shows a schematic view of the vacuum chamber in between the two vessels housing the printed circuit boards (PCBs) with the sample to be investigated, the trigger detector, and the radioactive source. A thin copper shield surrounds the PCB's and the source, in order to reduce thermal radiation. The two small interchangeable chip carriers are mounted on a larger PCB, the "motherboard", which is connected to the liquid nitrogen bath via a thermal bridge.

On the front side of the detector chip carrier are also placed a thermometer and the input FET of the pre-amplifier, which reads out the detector signal. A $100 \Omega$ heater is soldered on the back of the detector chip carrier. With this set-up it was possible to accurately monitor the temperature of the detector mount while preventing noise pick-up from the heater by the cold FET. Using a Digital Temperature Controller (SI 9650) it was possible to adjust and stabilise the temperature in the range between $78 \mathrm{~K}$ and $250 \mathrm{~K}$.

For the CCE measurements, the samples were exposed to a ${ }^{90} \mathrm{Sr}$ beta source. A silicon detector was used as a trigger to select minimum ionising particles (MIPs). The detector signals were read out by a charge amplifier, which had the cold FET as first front-end stage. The noise of this amplifier was about $1500 \mathrm{e}^{-}$FWHM. The signals were then shaped and recorded by an ADC. A typical charge spectrum is shown in Figure 3.

The recorded spectrum was fitted by a Landau distribution and the most probable value was determined. The CCE was calculated as the ratio of the pedestal subtracted most probable value to the one obtained with a non-irradiated sample of the same kind operated above full depletion. The absolute charge calibration is affected by a systematic error of $1.5 \%$ due to the 
uncertainty on the detector thickness. The errors shown in the plots of this paper are the statistical errors from the fit procedure.

\section{Current-voltage characteristics}

The current-voltage (I-V) characteristics of the detectors were measured using a Keithley 478 picoammeter. For measurements at $77 \mathrm{~K}$, the samples were cooled by direct immersion into a liquid nitrogen bath. The maximum bias voltage that could be applied was $500 \mathrm{~V}$, limited by the isolation of the detector mount. The sensitivity of the current measurement was about $1 \mathrm{nA}$, limited by the leakage of the bias voltage filtering capacitor.

Figure 4 shows the I-V characteristics at room temperature of the irradiated standard (left) and double-p (right) detectors. The symmetric characteristic of the double-p device shows a current of the same order of magnitude as the forward current of the $\mathrm{p}^{+} / \mathrm{n} / \mathrm{n}^{+}$detector. Before irradiation a maximum voltage of $80 \mathrm{~V}$ could be applied to the double-p detector due to back side injection (one junction is forward-biased at full depletion voltage).

Figure 5 shows the I-V characteristic of the irradiated double-p detector measured at $77 \mathrm{~K}$, for both polarities of the applied bias voltage. The steady state current is limited by the bulk resistivity and is below $1 \mathrm{nA}$. The same result is obtained in the case of the standard detector, both in reverse and in forward bias, in agreement with previous observations [3]. At this temperature, the detectors are characterized by a bulk material, which behaves like a resistor of a very high resistance value.

\section{CCE measurements}

For measuring the charge collection efficiency the following procedure was applied. After having installed the chip carriers in the cryostat, the vacuum chamber was closed and evacuated by means of a rotary pump. Typically, a pressure of $\sim 10^{-3}$ mbar was reached. The inner tank was then filled with liquid nitrogen, and the cooling down towards the desired temperature was started. The minimum sample temperature of $78 \mathrm{~K}$ could be reached in approximately one hour.

Before applying the desired bias voltage, the detector was kept at zero bias for at least fifteen minutes. The reason for this is that the bias voltage history plays a role in the time evolution of the signal amplitude at cryogenic temperatures for detectors rich in deep levels, like heavily irradiated silicon. This phenomenon was originally observed in germanium detectors and was called "detector polarisation" [13]. The charge spectrum was recorded just after the voltage turn-on, and then after several time intervals, in order to study the time 
evolution of the CCE. All CCE measurements were taken with two different shaping times: $1 \mu \mathrm{s}$ and $0.25 \mu \mathrm{s}$. Both shaping times gave the same CCE values within the uncertainties. In this paper only the results of measurements with a shaping time of $1 \mu$ s are shown.

Figure 6 shows the CCE of the irradiated double-p detector as a function of the bias voltage, when operated at three different temperatures. At $\mathrm{T}=200 \mathrm{~K}$ and at bias voltages larger than $+200 \mathrm{~V}$ or $-300 \mathrm{~V}$, the charge spectrum recorded by the oscilloscope was so noisy that it was not possible to perform a proper Landau fit. The plot clearly shows the expected symmetry of the sample for positive and negative applied bias voltage. The CCE increases with the absolute value of the applied bias voltage, and it does not seem to reach a plateau, indicating that higher values could be reached at higher voltages. The maximum CCE values are obtained for a temperature of $130 \mathrm{~K}$, in agreement with previous experimental observations on standard detectors [3]. This probably corresponds to the situation in which, at equilibrium, the fraction of filled radiation induced traps is such that the compensation of the space charge density, $N_{\text {eff }}$, is optimised. Below this temperature too many traps are filled and $N_{\text {eff }}$, which initially decreased in absolute value with temperature, increases again with a consequent decrease in the active volume for the same value of the applied bias voltage. Moreover, at lower temperatures signal is lost due to a strong increase in the trapping cross section. At the temperature of $130 \mathrm{~K}$ and with a $500 \mathrm{~V}$ applied bias, the 27000 electrons (most probable value) delivered by the sample when traversed by a MIP represents an unprecedented signal for a silicon detector irradiated to such a high dose.

Figure 7 shows the CCE at $\mathrm{T}=130 \mathrm{~K}$ as a function of the bias voltage, for measurements taken after several waiting time periods after voltage turn-on, for the (left) standard and (right) double-p detectors. The results obtained after 30 minutes are compatible, within the measurement error, with the ones obtained after 24 hours, and are thus considered as stable values. The standard detector shows a decay of CCE in time in the reverse bias case, while no time dependence is observed under forward bias. A similar behaviour was observed for all $\mathrm{p}^{+} / \mathrm{n} / \mathrm{n}^{+}$detectors that have previously been investigated [3]

In our case of the double-p detector, the CCE stable values are only a few percent lower than the ones obtained soon after voltage turn-on, in particular at higher bias voltages. Nevertheless they are systematically lower, indicating that there is some evolution in time of the CCE for both bias voltage polarities. Therefore, the device does not seem to have a classical "ohmic-like" behaviour. This is consistent with the early findings of double junction behaviour in highly irradiated $\mathrm{p}^{+} / \mathrm{n}^{+} \mathrm{n}^{+} \mathrm{Si}$ detectors $[14,15]$, with a minor junction (not very 
sensitive to the bias voltage) existing in the $\mathrm{p}^{+}$-side. In our case of a double-p detector, there could be two minor junctions existing on each side after irradiation. However, the CCE decrease is clearly less important than for the standard detector. It is worth stressing that the CCE of the standard detector is considerably lower than that of the double-p detector at all bias voltages.

In order to better understand the CCE dependence on time, the same data as in Figure 7 are plotted again in Figure 8, where the $\mathrm{CCE}$ at $\mathrm{T}=130 \mathrm{~K}$ is shown as a function of time after voltage turn-on. The data of the standard detector (left) refers to reverse bias operation. It is clear that the $\mathrm{CCE}$ decrease in time is stronger at lower bias voltages, and it takes place in the first few minutes. A similar trend can be seen for the double-p detector (right), although the amount of decay in time is smaller. Moreover, while for a bias voltage below or equal to $200 \mathrm{~V}$ the CCE shows a decay of the order of $10 \%$, it is basically constant in time at $500 \mathrm{~V}$.

Another important feature can be extracted from Figure 9, which shows the CCE of the irradiated double-p detector as a function of time after voltage turn-on at three different temperatures. The bias voltage was always set at $200 \mathrm{~V}$. It is clear from this plot that the operating temperature has an influence on the time evolution of the CCE. In particular, the decay in time is higher at lower temperatures. At $\mathrm{T}=200 \mathrm{~K}$ the CCE is practically constant in time. This is probably due to the fact that the higher steady state current provides a larger number of carriers, which makes the equilibrium being reached in a shorter time interval.

\section{Conclusions}

We have found that a double-p silicon detector irradiated to $1 \times 10^{15} \mathrm{n} / \mathrm{cm}^{2}$ shows the unprecedented CCE of $\sim 80 \%$ when operated at $130 \mathrm{~K}$ and at a $500 \mathrm{~V}$ applied bias. For a $400 \mu \mathrm{m}$ thick detector traversed by a minimum ionising particle, this corresponds to a signal of about 27000 electrons. The sample shows the expected symmetry with respect to the applied bias voltage, before and after irradiation and at any temperature used in this study. The CCE of the sample follows the trend already studied for standard irradiated silicon detectors, showing a dramatic recovery of the CCE at cryogenic temperatures, which peaks at $130 \mathrm{~K}$.

The double-p detector also features a small but undesired time dependence of the CCE, which decreases with increasing bias voltage and increasing temperature. Apparently the device does not have a completely "ohmic-like" behaviour as expected after irradiation and type inversion due to the double junction effect. Nevertheless, the CCE decay is considerably smaller than for standard detectors operated in reverse bias. Symmetric detectors can thus be 
considered as a solution to the problem of the time evolution of the CCE. The only drawback of such a detector consists of the fact that, before irradiation, particular care must be given to the applied bias, because there is no protection against backside injection.

\section{Acknowledgements}

This research was supported by: the Schweizer Nationalfonds zur Förderung der wissenschaftlichen Forschung (FORCE); the Stiftung zur Förderung der wissenschaftlichen Forschung an der Universität Bern; the Ministry of Science and Technology of the Republic of Slovenia. The work of two of the authors (Z. Li and B. Dezillie) was supported by the U.S. Department of Energy, Contract Number DE-AC02-98CH10886. One of the authors (S. R. H. Devine) acknowledges financial support from the Particle Physics and Astronomy Research Council (PPARC) and also acknowledges RAL for the CASE Award. We are grateful to Prof. Klaus Pretzl and Prof. Peter Sonderegger for their interest and continuous support of this work. The CERN Microelectronics Workshop is greatly acknowledged for technical support.

\section{References}

[1] V.G. Palmieri et al., Nucl. Instr. and Meth. Phys. Res. A413 (1998) 475

[2] K. Borer et al. (RD39 Collab.), CERN/LHCC 98-27, DRDC P53 Add. 1 (1998)

[3] K. Borer et al. (RD39 Collab.), Nucl. Instr. and Meth. A440 (2000) 5

[4] K. Borer et al., Nucl. Instr. and Meth. A440 (2000) 17

[5] RD48 Collaboration, CERN/LHCC 2000-009, LEB Status Report/RD48 (1999)

[6] For a review see: Proc. of the $1^{\text {st }}$ Int. Conf. on Radiation Effects on Semic. Materials, Detectors and Devices, Nucl. Instr. and Meth. Phys. Res. A388 (1997)

[7] L. Beattie et al., Nucl. Instr. and Meth. A412 (1998) 238

[8] B. Dezillie, V. Eremin and Z. Li, IEEE Trans. on Nucl. Sci. 46-3 (1998) 221

[9] B. K. Jones, J. Santana and M. McPherson, Nucl. Instr. and Meth. A395 (1997) 81

[10] A. Chilingarov and T. Sloan, Nucl. Instr. and Meth. A399 (1997) 35

[11] A. M. Ougouag et al., IEEE Trans. on Nucl. Sci. NS-37 (1990) 2219

[12] P. J. Griffin et al., pre-print SAND92-0094, Sandia Natl. Lab., 1993

[13] V. Eremin et al., Sov. Phys. \& Techn. of Semiconductors 8 (1974) 1157

[14] Z. Li and H.W. Kraner, J. Electron. Mater. 21 (7) (1992) 701

[15] D. Menichelli et al., Nucl. Instr. and Meth. A426 (1999) 135 
Before irradiation
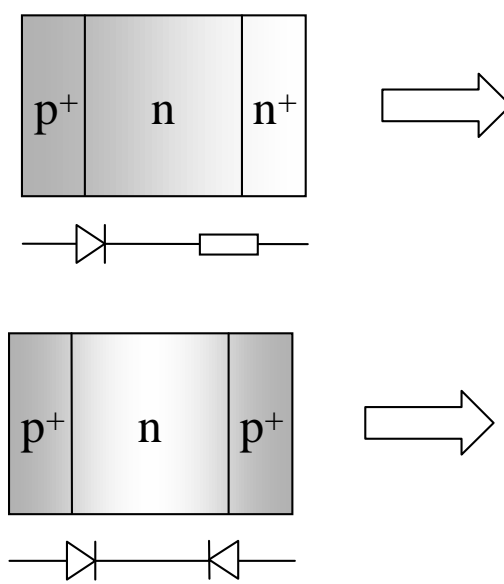

After irradiation
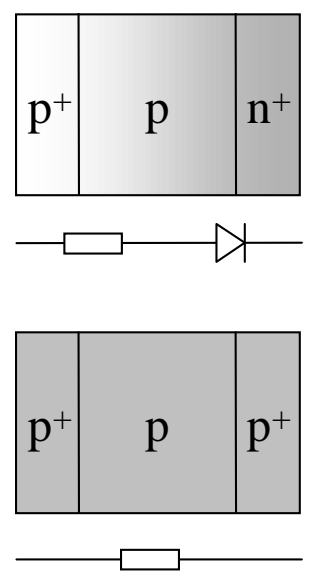

Figure 1. Schematic representation of the type inversion after irradiation for standard (top) and double-p (bottom) silicon detectors.

Front view

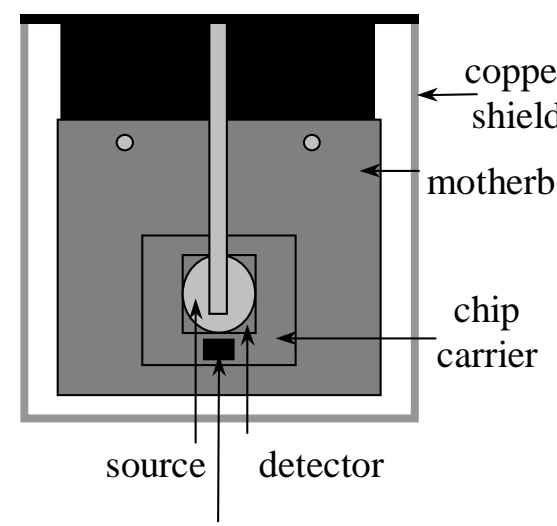

thermometer
Side view

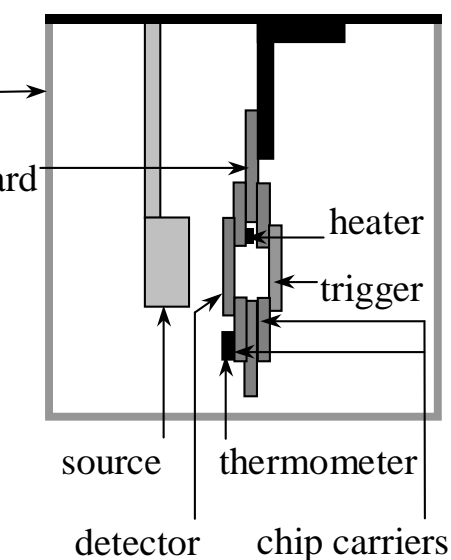

Figure 2. Close view of the vacuum chamber of the cryostat. The PCB's have a hole at the position of the detector and trigger in order to minimise the amount of material traversed by the electrons emitted by the source. 


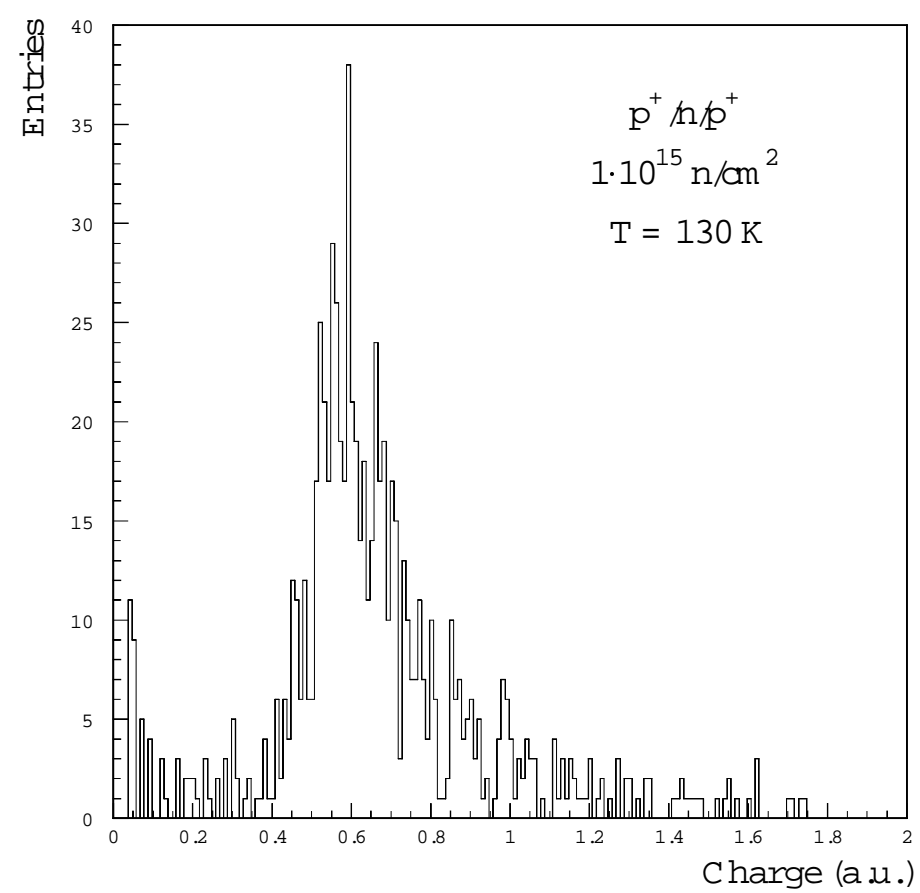

Figure 3. Example of a recorded charge spectrum. It refers to the irradiated double-p sample operated at $130 \mathrm{~K},-100 \mathrm{~V}$ bias, soon after voltage turn-on. The shaping time was $1 \bullet \mathrm{s}$.
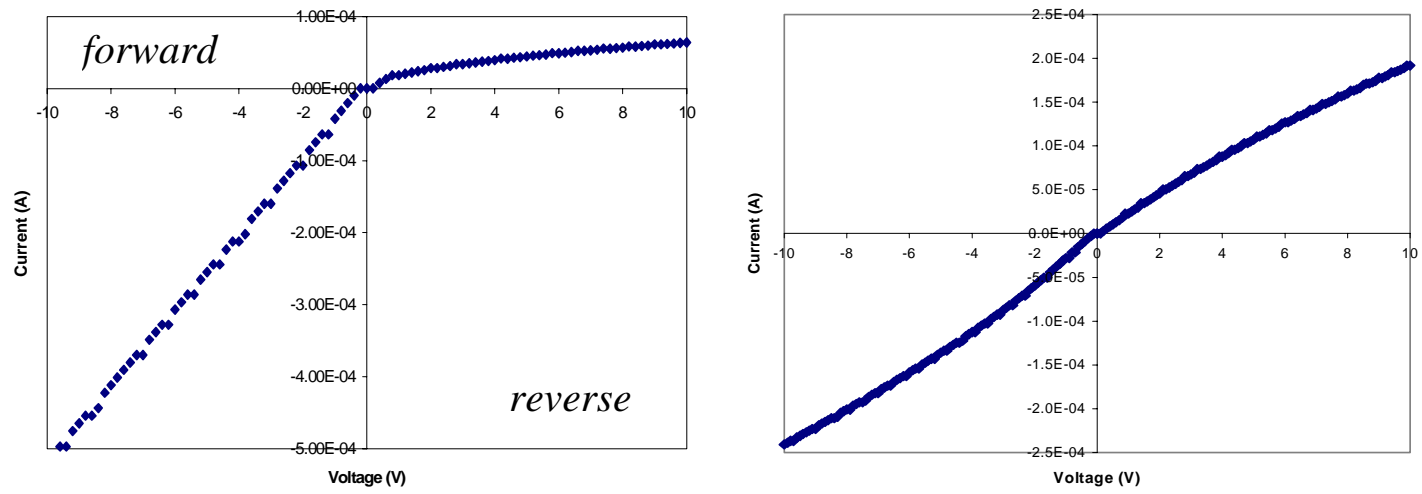

Figure 4. I-V characteristic at room temperature of the irradiated standard (left) and double-p (right) silicon detectors. The steady state current of the double-p device is of the same order of magnitude as the forward current of the standard detector. 


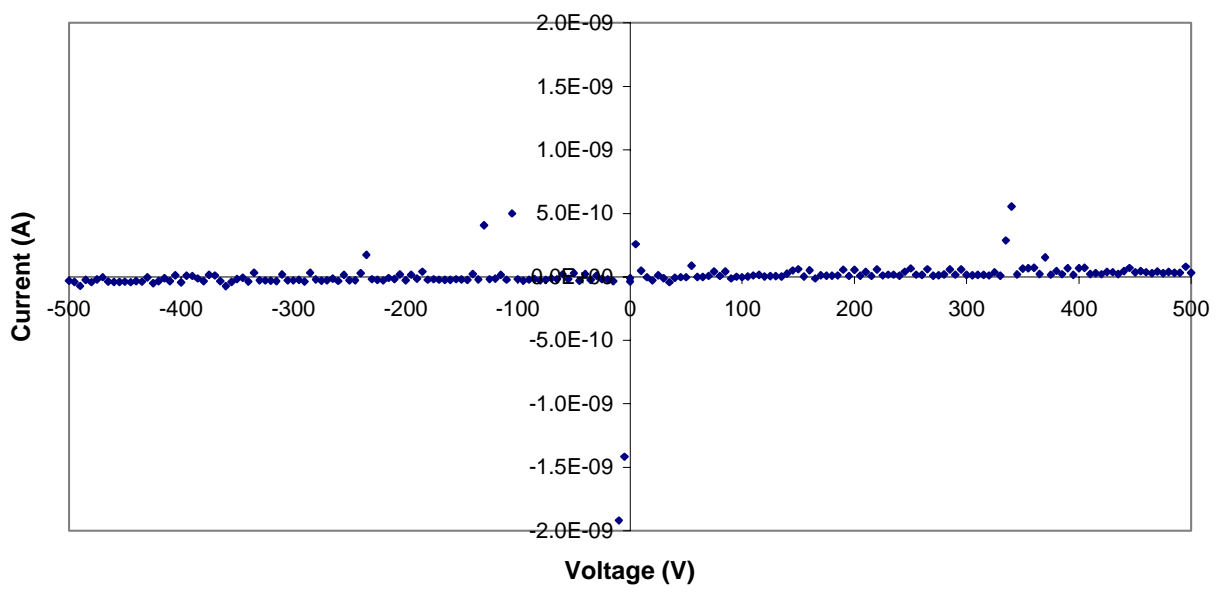

Figure 5. I-V characteristic of the irradiated double-p detector at $T=77 \mathrm{~K}$. At this temperature, the steady state current is below $1 \mathrm{nA}$ also in the case of the standard detector, whatever the polarity of the bias voltage is.

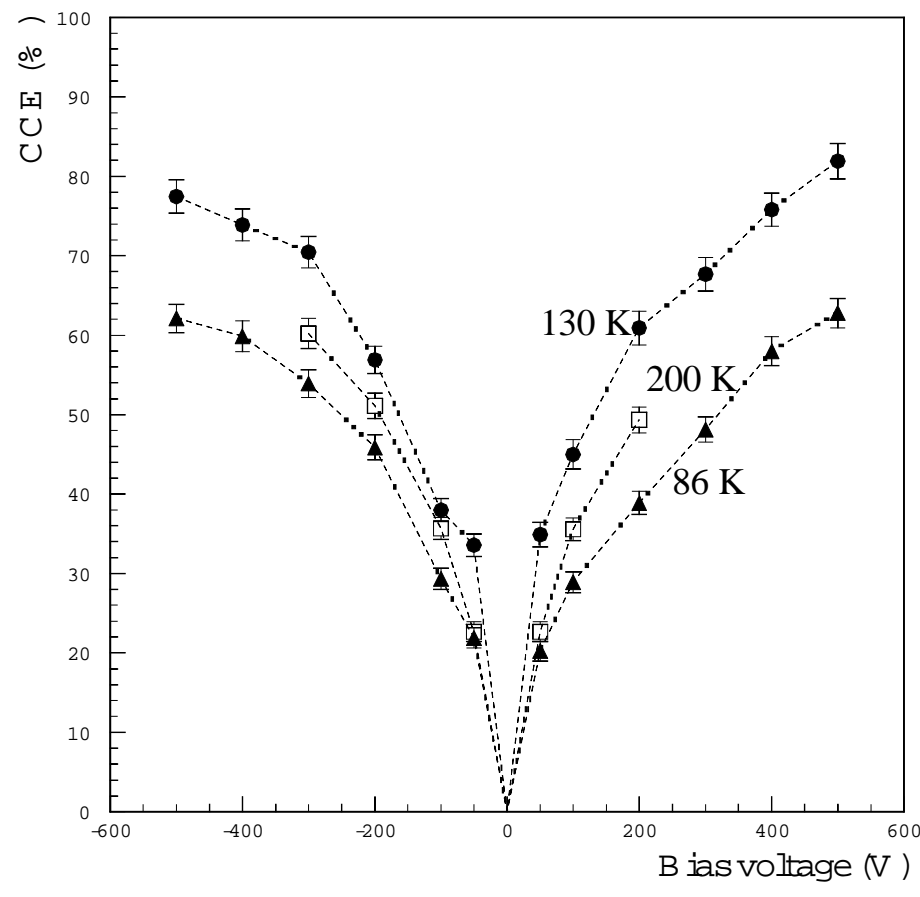

Figure 6. CCE of the double-p detector as a function of the bias voltage, at three different operating temperatures. The data points were measured 30 minutes after voltage turn-on. 

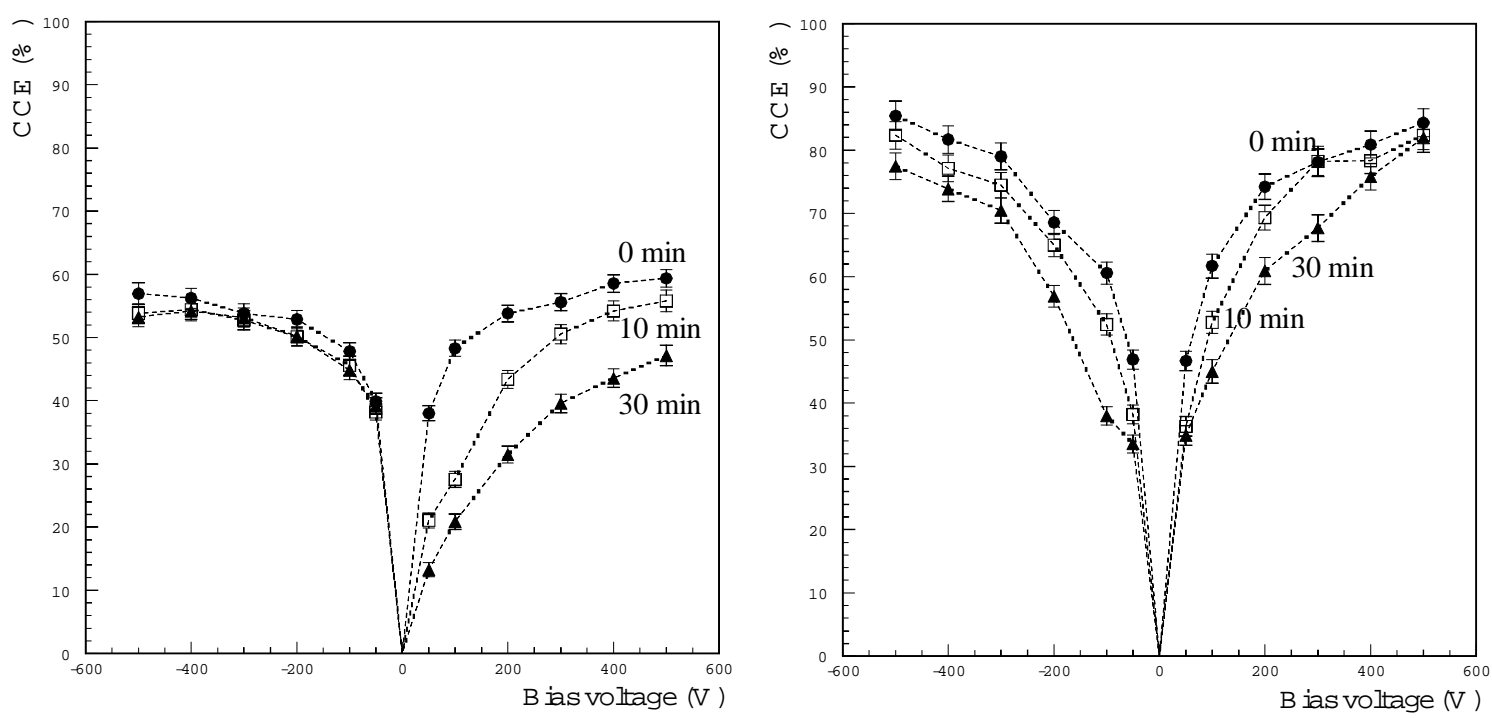

Figure 7. CCE as a function of the bias voltage at several time intervals after voltage turn-on for the standard (left) and the double-p (right) detectors. In both cases the temperature was $130 \mathrm{~K}$.
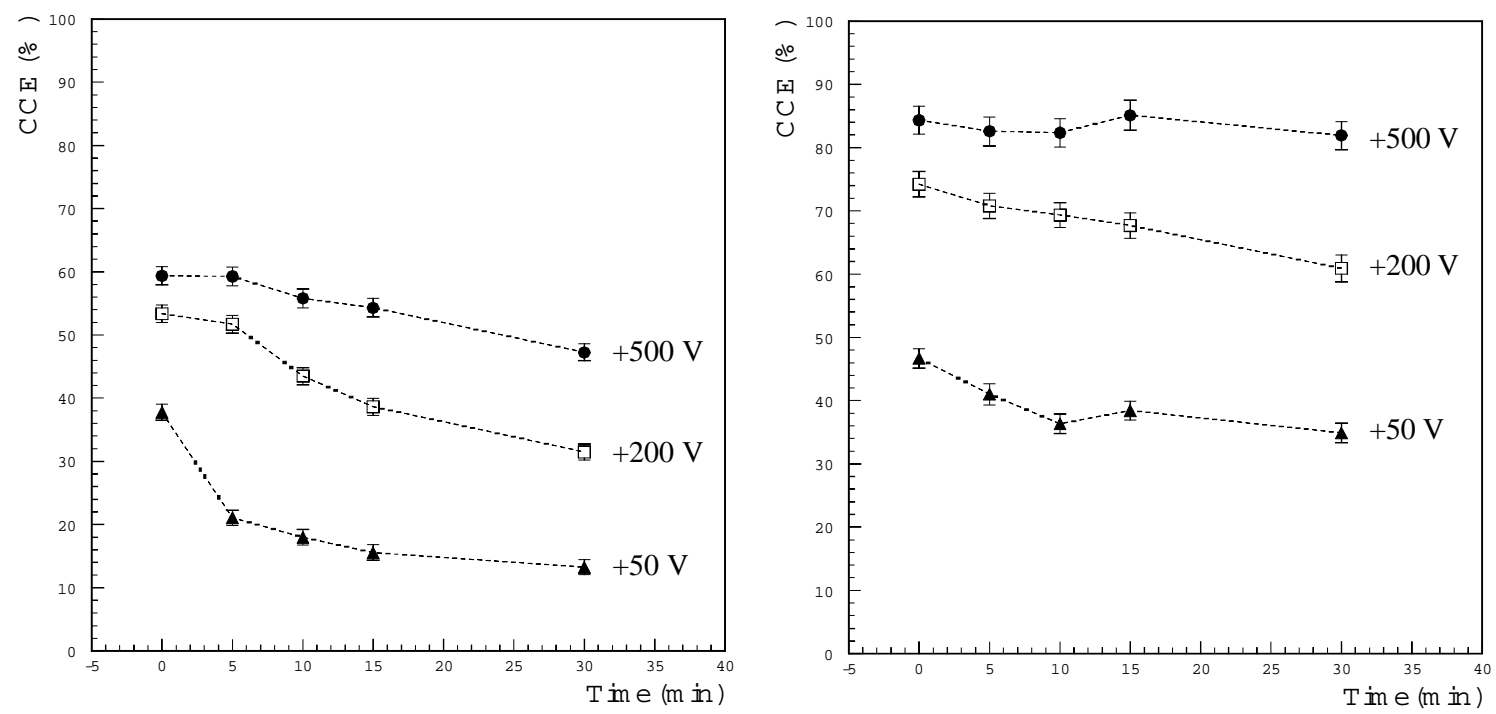

Figure 8. CCE as a function of the time elapsed after voltage turn-on for the standard (left) and the double-p (right) detectors. The measurements were taken at $130 \mathrm{~K}$. The CCE decay in time is stronger at lower bias voltages. 


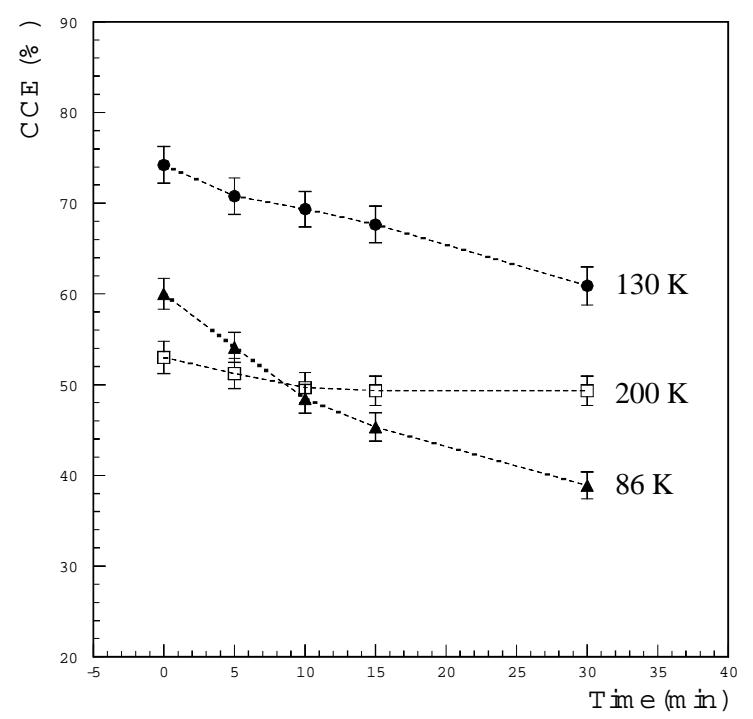

Figure 9. CCE of the double-p detector as a function of the time elapsed after voltage turn-on. The bias voltage was set to $200 \mathrm{~V}$. The CCE decrease in time is stronger at lower temperatures. 